\title{
The New Method of Finding of Optimal Regularization Parameter for Potential Field Transformation
}

\author{
Pyankov Valentin, Rublev Alexey
}

\begin{abstract}
We have investigated the morphology of the negative regional gravity anomaly with maximal value near 300 mGl at different altitudes (Sierra Nevada Batholith). This gravity anomaly spatially coincides with the structures of San Andreas and Calaveras faults (20-55 degrees S and 90-140 degrees W). A summary of the geological-geophysical data described the structure near transform fault system San Andreas and a thin structure of the master fault (San Andreas Fault), which accommodated more than a half of the relative shear movements of the Pacific and North American lithospheric plates, are used. In this paper we apply the technique of filtration of the long- and short-wave components of the amplitude spectrum of anomalies, using numerical methods of field simulation at various altitudes. For creation the multilayer gravity model of the Earth crust we need to know the field morphology of different earth layers. For this purpose we have investigated the optimal regularization parameters for gravitational field transformation.
\end{abstract}

Keywords - gravity field, regularization parameter, upward and downward continuation

\section{INTRODUCTION}

Estimates of lithospheric densities are unreliable when they are based only on modeling the observed gravity field because of the intrinsic non-uniqueness of gravity data inversions. To enhance the accuracy of gravity models, a sufficient quantity of a priori seismic, geophysical, geological, and petrological constraints are needed. Also, stronger lateral density contrasts within both the crust and the mantle, and more pronounced depth undulations of density boundaries yield better conditions for density modeling. These conditions are satisfied in southern California, and we present transformed gravity field near San Andreas Fault, the geophysics of which are already well studied.

The authors are with the Bulashevich Institute of Geophysics UB of RAS Yekaterinburg, Russia
The construction of regional geological-geophysical models, their refinement, and petrographic specification are based on the method of gravitational modeling. The central problem of the method is the choice of maximally stable geologically informative solutions of the inverse gravity problem from the set of possible equivalents. It is known with certainty that the inverse gravity problem is incorrect, i.e., its solution is nonunique and critically dependent on the initial data. In this paper we attempt to construct threedimensional geological-geophysical models of practical interest with the help of the joint interpretation of gravity and seismic data in terms of the adopted conceptions. The solution of the inverse problem is based on the method of local correction described by Martyshko and Prutkin [1]. In contrast to other methods (such as the trial-and-error method and the method of the inversion of the forward problem operator), the iteration method of local corrections possesses a greater algorithmic simplicity and ensures a more rapid convergence of the solution. The method of the joint interpretation proposed in the paper makes it possible to narrow the class of possible solutions and makes the algorithm of their construction stable with respect to noise. Our technique uses a new approach for the construction of a few structural boundaries in which components considered as the fields of the boundaries under study are extracted from the observed field. For creation the multilayer gravity model of the Earth crust we need to know the field morphology of different earth layers [2]. We have studied the single gravity anomaly of Sierra Nevada Batholith (Figure 1) to develop a methodology for determining the optimal regularization parameters.

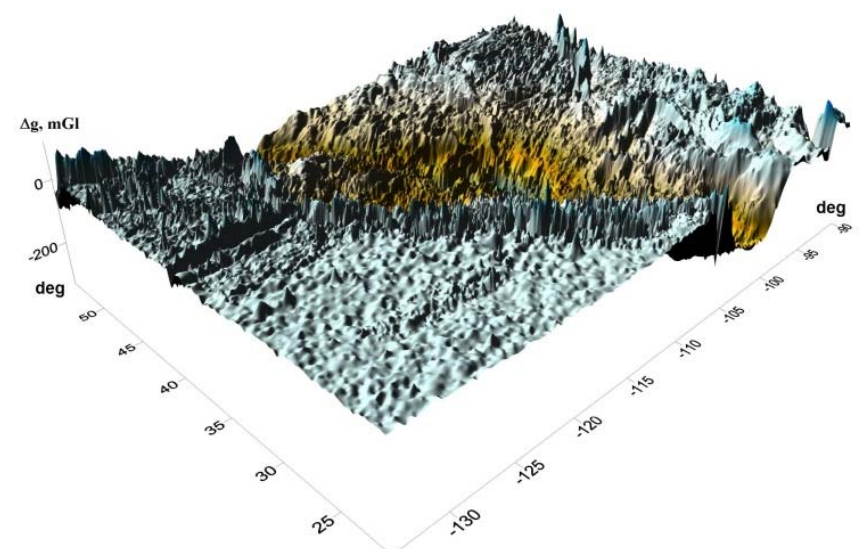

Fig. 1. The gravity field of Sierra Nevada Batholith. 


\section{METHOD}

To divide the long- and short-wave components of the amplitude spectrum of anomalies, geophysicists utilize numerical methods of field simulation at various altitudes. In our paper we investigate gravitational field near transection of San-Andreas and Calaveras faults $(\mathrm{S}=4029 \times 3646 \mathrm{~km})$. The software implementing parallel algorithms of altitude transformations are developed using the MVAPICH2 library in Fortran programming language. The parallel algorithms of the height transforms based on the gradient type iterative methods have been designed and numerically implemented on Uran supercomputer at the Institute of Mathematics and Mechanics of the Ural Branch of the Russian Academy of Sciences [7]. The Uran supercomputer is a cluster of multiprocessor systems, each computing unit of which is based on two 4-core Intel QuadCore Xeon processors with a speed of $3 \mathrm{GHz}$ and a $16 / 32$ $\mathrm{Gb}$ cache. Overall, 1664 computing cores and $3584 \mathrm{~Gb}$ cache memory are available to the user. The parallel algorithms for recalculation were implemented for $512 \mathrm{x}$ 512 points of the mesh.

We introduce the rectangular Cartesian coordinates with axis $\mathrm{Z}$ pointing downwards and plane XOY coinciding with the surface of the observations. Transformation of the gravity field $\left.U(x, y, z)\right|_{z=0}$ measured on the Earth's surface area $D=\left\{(x, y) \in R^{2}: a \leq x \leq b, c \leq y \leq d\right\}$ upwards to the level $z=-H$ is made by the Poisson formula:

$$
\begin{aligned}
& U(x, y,-H)= \\
& =\frac{1}{2 \pi} \int_{-\infty-\infty}^{+\infty} \int_{[-\infty}^{+\infty} \frac{-H}{\left[\left(x-x^{\prime}\right)^{2}+\left(y-y^{\prime}\right)^{2}+H^{2}\right]^{3 / 2}} U\left(x^{\prime}, y^{\prime}, 0\right) d x^{\prime} d y^{\prime} .
\end{aligned}
$$

For recalculating the gravity field downwards to depth $z$ $=H$ and finding the values $\bar{U}(x, y,-H)$, we solve Fredholm equation of the first kind:

$$
\begin{aligned}
& K u \equiv \frac{1}{2 \pi} \int_{-\infty}^{+\infty} \int_{-\infty}^{+\infty} \frac{2 H}{\left[\left(x-x^{\prime}\right)^{2}+\left(y-y^{\prime}\right)^{2}+4 H^{2}\right]^{3 / 2}} \times \\
& \times \bar{U}\left(x^{\prime}, y^{\prime}, H\right) d x^{\prime} d y^{\prime}=U(x, y,-H) .
\end{aligned}
$$

After discretization of the integrated equation (2), we obtain a system oflinear algebraic equations. (2) belongs to the class of ill-posed problems; therefore, its solution implies regularization by a shift [3].

$$
(K+\alpha I) u=U,
$$

where $\alpha$ is the regularization parameter.

To solve (3), we used the iteratively regularized method of simple iteration:

$$
u^{k+1}=u^{k}-\frac{1}{\lambda_{\max }}\left[(K+\alpha I) u^{k}-U\right]
$$

where $\lambda_{\max }$ is the maximal eigenvalue of matrix $K+\alpha I$ (symmetric case).

The condition of iteration stop is the satisfied inequality

$$
\frac{\left\|K u^{k}-\bar{U}\right\|}{\|\bar{U}\|}<\varepsilon
$$

with a sufficiently small $\varepsilon$.

\section{SELECTING THE REGULARIZATION PARAMETER}

In our paper we investigate the optimal regularization parameters for gravitational field transformation. The downward continuation procedure is an ill-posed problem so the regularization is applied. For selecting the regularization parameter $\alpha$, we used the results of the transformation of the gravity anomalous field (Sierra Nevada Batholith). The problem of this paper to divide the long- and short-wave components of the amplitude spectrum of anomalies, geophysicists utilize numerical methods of field simulation at various altitudes. For this purpose, we used to calculate a set of gravity field values recalculated to a height of $-H$ and the corresponding field recalculated down on $2 \mathrm{H}$ and related to the level of the earth's surface. The calculations were made for the altitudes $H=10,20,30,40,50,60,70,100,150$ and $200 \mathrm{~km}$. Recalculation to the Earth's surface is given for the regularization parameters $\alpha$ from 0.01 to 0.86 . The difference between the transformations of the gravity field to a depth of $70 \mathrm{~km}$ for different regularizes ( $\alpha$ equal 0.86 and 0.01 ) is shown in Figure 2.

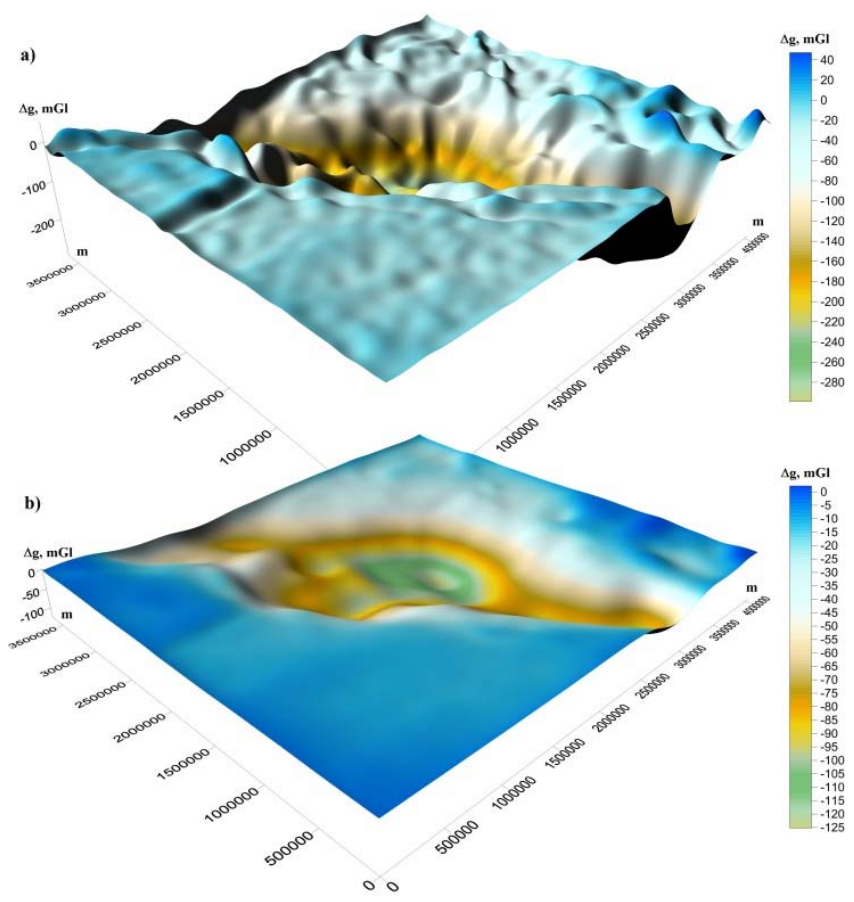

Fig. 2. Transformations of the gravity field to a depth of $70 \mathrm{~km}$ for different regularization parameters: a) $\alpha=0.86$, b) $\alpha=0.01$.

Next, for set of regularization parameters $\alpha$ value of the difference of fields recalculated to a height $-H$ and related to the level of the earth's surface was determined. We have found such a value regularizer $\alpha$, where the differences of squares of deviations of the corresponding field values have reached its minimum. In addition, the correlation coefficients of the difference of fields with the field, converted to a height $-H$ were determined. The optimum values of the regularization parameter $\alpha$ are shown in the graph, and approximated by a polynomial of degree 4 (Figure 3 ). 


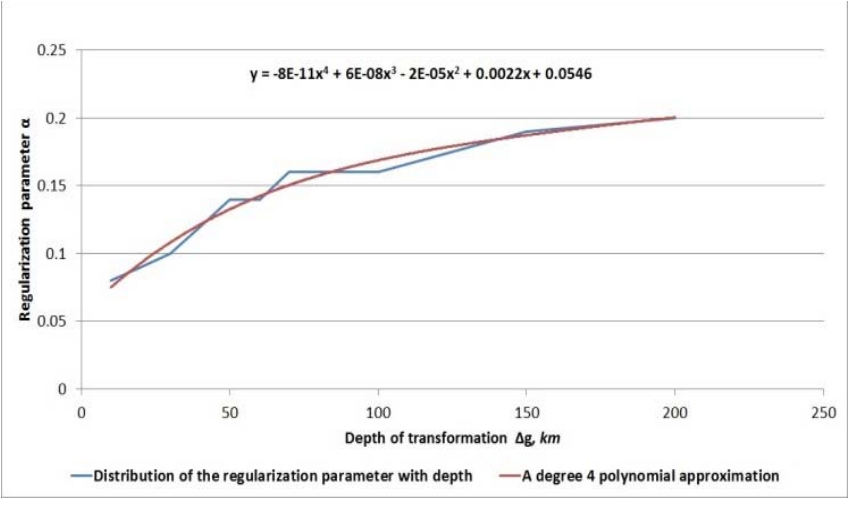

Fig. 3. Distribution of the regularization parameter with depth.

\section{CONCLUSIONS}

Our investigations have shown:

1. Analysis of the morphology of the regional single negative gravity anomaly Sierra Nevada at different altitudes characterizes general law of the regularization parameter distribution. That is narrow range of its values: $0.07-0.2$.

2. The small variations of the regularization parameter reduce to big variations of transformed gravity field.

3. The authors suggest to use the results of this research for solving the gravity inverse problem for multilayered earth crust on the Arctic Urals.

\section{ACKNOWLEDGMENT}

We thank Romanyuk T.V. for providing the actual gravity data.

\section{REFERENCES}

[1] P.S. Martyshko and I.L. Prutkin, "Technology for separating the gravity sources by the depth”, Geofizicheskiy Zhurnal, vol. 25, № 3, pp. 159-168, 2003.

[2] P.S. Martyshko, I.V. Ladovskii and A.G. Tsidaev, "Construction of regional geophysical models based on the joint interpretation of gravity and seismic data", Izvestiya, Physics of the Solid Earth, vol. 46, № 11, pp. 931-942, 2010. DOI: 10.1134/S1069351310110030

[3] M.M. Lavrentiev, "Some Improperly Posed Problems of Mathematical Physics", Springer, New York, 1967, 76 p. DOI $10.1007 / 978-3-642-88210-4$ 